\title{
NICE GROUP STRUCTURE ON THE ELEMENTARY ORBIT SPACE OF UNIMODULAR ROWS
}

\author{
MANOJ K. KESHARI AND SAMPAT SHARMA
}

2020 Mathematics Subject Classification:13C10, 19D45, 19G12

Keywords: Unimodular row, nice group structure

\begin{abstract}
Let $R$ be an affine algebra of dimension $d \geq 5$ over $\overline{\mathbb{F}}_{p}$ with $p>3$. Then the group structure on $\mathrm{Um}_{d}(R) / \mathrm{E}_{d}(R)$ is nice.

(2) Let $R$ be a commutative noetherian ring of dimension $d \geq 2$ such that $\mathrm{E}_{d+1}(R)$ acts transitively on $\operatorname{Um}_{d+1}(R)$. Then the group structure on $\mathrm{Um}_{d+1}(R[X]) / \mathrm{E}_{d+1}(R[X])$ is nice.
\end{abstract}

\section{INTRODUCTION}

All the rings are assumed to be commutative noetherian with unity 1.

Let $n \geq 3$ be an integer and $R$ be a ring with stable dimension $\leq 2 n-4$. It was proved by van der Kallen [10] that the orbit space $\operatorname{Um}_{n}(R) / \mathrm{E}_{n}(R)$ has an abelian group structure. Here $\operatorname{Um}_{n}(R)$ and $\mathrm{E}_{n}(R)$ denote the set of unimodular rows and the group generated by elementary matrices (definition 2.1). If $v, w \in \operatorname{Um}_{n}(R)$, then in view of [11, Lemma 3.2], the classes $[v]$ and $[w]$ can be expressed as $[v]=\left[\left(x_{1}, v_{2}, \ldots, v_{n}\right)\right]$ and $[w]=\left[\left(v_{1}, v_{2}, \ldots, v_{n}\right)\right]$. Then their product is defined in [10, Lemma 3.5 (i)] as

$$
\left[\left(x_{1}, v_{2}, \ldots, v_{n}\right)\right] *\left[\left(v_{1}, v_{2}, \ldots, v_{n}\right)\right]=\left[\left(v_{1}\left(x_{1}+w_{1}\right)-1,\left(x_{1}+w_{1}\right) v_{2}, v_{3}, \ldots, v_{n}\right)\right]
$$

where $w_{1} \in R$ is such that $v_{1} w_{1}=1$ modulo $\left(v_{2}, \ldots, v_{n}\right)$. We say that this group operation is nice if it satisfies

$$
\left[\left(x_{1}, v_{2}, \ldots, v_{n}\right)\right] *\left[\left(v_{1}, v_{2}, \ldots, v_{n}\right)\right]=\left[\left(x_{1} v_{1}, v_{2}, \ldots, v_{n}\right)\right] .
$$

Let $C=\mathbb{R}\left[x_{0}, x_{1}, \ldots, x_{d}\right] /\left(x_{0}^{2}+x_{1}^{2}+\cdots+x_{d}^{2}-1\right)$ be the coordinate ring of the real $d$-sphere $S^{d}$ and $R=$ $T^{-1} C$, where $T$ is the multiplicative set of polynomial functions that do not have any zero on $S^{d}$. Then the group structure on $\mathrm{Um}_{d+1} / \mathrm{E}_{d+1}(R)$ is not nice [22, Example 2.2(c)]. We state some results where group structure on $\mathrm{Um}_{n}(R) / \mathrm{E}_{n}(R)$ is nice.

(1) If $R$ is a smooth affine algebra of dimension $d \geq 3$ over a perfect field $k$ with c.d.2 $(k) \leq 2$, then Fasel [5. Theorem 2.1] proved that the group structure on $\operatorname{Um}_{d+1}(R) / \mathrm{E}_{d+1}(R)$ is nice.

(2) Garge and Rao [6, Theorem 3.9] removed smoothness assumption in Fasel's result under the additional assumption that $\operatorname{char}(k) \neq 2$ and c.d. $(k) \leq 1$.

(3) If $R$ is a smooth affine algebra of dimension $d \geq 3$ over an algebraically closed field, then Gupta, Garge and Rao [7, Theorem 6.8] proved that the group structure on $\mathrm{Um}_{d}(R) / \mathrm{E}_{d}(R)$ is nice. 
(4) If $R$ is a local ring of dimension $d$ with $2 R=R$, then Garge and Rao [6, Theorem 5.1] proved that the group structure on $\operatorname{Um}_{d+1}(R[X]) / \mathrm{E}_{d+1}(R[X])$ is nice.

In [17, Proposition 3.5], Jose and Rao proved that if $R$ is a reduced affine algebra of dimension $d \geq 2$ over an algebraically closed field $k$, then the group structure on $\operatorname{Um}_{d+1}(R) / \mathrm{E}_{d+1}(R)$ is nice. If $\left(c_{0}, c_{1}, \ldots, c_{d}\right) \in$ $\mathrm{Um}_{d+1}(R)$, then by Swan's version of Bertini theorem [19], we may assume that $R /\left(c_{1}, \ldots, c_{d}\right)$ is a finite product of $k$. Thus $c_{0}=d_{0}^{2} \bmod \left(c_{1}, \ldots, c_{d}\right)$. Let us see that this implies that the group structure on $\mathrm{Um}_{d+1}(R) / \mathrm{E}_{d+1}(R)$ is nice. Let $v=\left(a_{0}, a_{1}, \ldots, a_{d}\right), w=\left(a_{0}^{\prime}, a_{1}, \ldots, a_{d}\right) \in \mathrm{Um}_{d+1}(R)$. Then there exist $b_{0}, b_{0}^{\prime} \in R$ such that

$$
[v]=\left[\left(b_{0}^{2}, a_{1}, \ldots, a_{d}\right)\right] \text { and }[w]=\left[\left(b_{0}^{\prime 2}, a_{1}, \ldots, a_{d}\right)\right] .
$$

Thus $a_{0} a_{0}^{\prime}=b_{0}^{2} b_{0}^{\prime 2} \bmod \left(a_{1}, a_{2}, \ldots, a_{d}\right)$. Therefore,

$$
[v] *[w]=\left[\left(b_{0}^{2} b_{0}^{\prime 2}, a_{1}, \ldots, a_{d}\right)\right]=\left[\left(a_{0} a_{0}^{\prime}, a_{1}, \ldots, a_{d}\right)\right]
$$

by [10, Lemma 3.5(v)]. If $R$ is an affine algebra of dimension $d \geq 3$ over $\overline{\mathbb{F}}_{p}$, then Jose and Rao [17, Proposition 3.5] states that the same proof will show that $\operatorname{Um}_{d}(R) / \mathrm{E}_{d}(R)$ has a nice group structure. We observe that if $v=\left(a_{0}, a_{1}, \ldots, a_{d-1}\right) \in \mathrm{Um}_{d}(R)$, then $R /\left(a_{1}, \ldots, a_{d-1}\right)$ is one dimensional. So it is not a finite product of $\overline{\mathbb{F}}_{p}$ and hence we do not have $a_{0}=b_{0}^{2} \bmod \left(a_{1}, \ldots, a_{d-1}\right)$. Therefore their proof does not work in this case. We revisit the method of Garge and Rao [6] and prove the following results.

Theorem 1.1. Let $R$ be an affine algebra of dimension $d \geq 5$ over $\overline{\mathbb{F}}_{p}$ with $p>3$. Then the group structure on $\mathrm{Um}_{d}(R) / \mathrm{E}_{d}(R)$ is nice.

Theorem 1.2. Let $R$ be a ring of dimension $d \geq 2$ such that $\mathrm{E}_{d+1}(R)$ acts transitively on $\operatorname{Um}_{d+1}(R)$. Then the group structure on $\mathrm{Um}_{d+1}(R[X]) / \mathrm{E}_{d+1}(R[X])$ is nice.

The above result applies to following two cases where $\mathrm{E}_{d+1}(R)$ acts transitively on $\operatorname{Um}_{d+1}(R)$ : (i) Jacobson radical of $R$ has height $\geq 1$, (ii) $R$ is an affine algebra over $\overline{\mathbb{F}}_{p}$.

Let $R$ be a ring with $\operatorname{sdim}(R) \leq 2 n-4$ and $n \geq 3$. Then van der Kallen [10, Theorem 4.1] has shown that the group $\operatorname{Um}_{n}(R) / \mathrm{E}_{n}(R)$ is the universal weak $n$-Mennicke symbol group $\mathrm{WMS}_{n}(R)$. When the group strucutre on $\operatorname{Um}_{n}(R) / \mathrm{E}_{n}(R)$ is nice, the universal weak $n$-Mennicke symbol group $\mathrm{WMS}_{n}(R)$ coincides with universal $n$-Mennicke symbol group $\operatorname{MS}_{n}(R)$.

\section{PRELIMINARIES}

Definition 2.1. Let $R$ be a ring and $I$ be an ideal of $R$.

- A vector $v=\left(a_{1}, \ldots, a_{r}\right) \in R^{r}$ is unimodular if there exist $\left(b_{1}, \ldots, b_{r}\right) \in R^{r}$ with $\langle v, w\rangle=$ $\sum_{i=1}^{r} a_{i} b_{i}=1$. The set of unimodular rows in $R^{r}$ is denoted as $\operatorname{Um}_{r}(R)$. Let $e_{i}=(0, \ldots, 1, \ldots, 0) \in$ $\mathrm{Um}_{r}(R)$, where 1 is at the $i^{\text {th }}$ place. The set $\operatorname{Um}_{r}(R, I)$ consist of unimodular vectors $v \in \operatorname{Um}(R)$ which are congruent to $e_{1}$ modulo $I$.

- The group $\mathrm{E}_{r}(R)$ is generated by elementary matrices $e_{i j}(\lambda)=\mathrm{I}_{r}+\lambda \mathrm{E}_{i j} \in \mathrm{GL}_{r}(R)$, where $\lambda \in R$ and for $i \neq j, \mathrm{E}_{i j} \in \mathrm{M}_{r}(R)$ whose $(i, j)^{t h}$ entry is 1 and all other entries are zero. Here $\mathrm{I}_{r} \in \mathrm{GL}_{r}(R)$ is identity matrix. The group $\mathrm{E}_{r}(R)$ acts on $\operatorname{Um}_{r}(R)$ by right multiplication, i.e. $v \cdot \varepsilon:=v \varepsilon$. We say $v \sim w$ if there exists an $\varepsilon \in E_{r}(R)$ such that $v=w \varepsilon$. One can check that $\sim$ is an equivalence relation. 
Let $\operatorname{Um}_{r}(R) / \mathrm{E}_{r}(R)$ denote the set of orbits of this action. The equivalence class of $v \in \operatorname{Um}_{r}(R)$ is denoted by $[v]$.

- Let $\psi_{1}=\left(\begin{array}{cc}0 & 1 \\ -1 & 0\end{array}\right)$ and $\psi_{n}=\psi_{n-1} \perp \psi_{1}$ for $n \geq 2$. Let $\sigma_{n}$ be the permutation of $\{1, \ldots, 2 n\}$ given by $\sigma_{n}(2 i)=2 i-1$ and $\sigma_{n}(2 i-1)=2 i$. The symplectic group $\operatorname{Sp}_{2 m}(R)$ is the subgroup of $\mathrm{GL}_{2 m}(R)$ consisting of all $\alpha \in \mathrm{GL}_{2 m}(R)$ such that $\alpha^{t} \psi_{m} \alpha=\psi_{m}$.

The elementary symplectic group $\operatorname{ESp}_{2 m}(R)$ is the subgroup of $\operatorname{Sp}_{2 m}(R)$ generated by elementary symplectic matrices

$$
s e_{i j}(z)= \begin{cases}I_{2 m}+z \mathrm{E}_{i j}, & \text { if } i=\sigma_{m}(j) \\ I_{2 m}+z \mathrm{E}_{i j}-(-1)^{i+j} z \mathrm{E}_{\sigma_{m}(j) \sigma_{m}(i)}, & \text { if } i \neq \sigma_{m}(j)\end{cases}
$$

for $1 \leq i \neq j \leq 2 m$ and $z \in R$.

- Let $\mathrm{GL}_{n}(R, I)$ denote the kernel of the canonical mapping $\mathrm{GL}_{n}(R) \longrightarrow \mathrm{GL}_{n}(R / I)$ and $\mathrm{SL}_{n}(R, I)=$ $\mathrm{SL}_{n}(R) \cap \mathrm{GL}_{n}(R, I)$.

- The group $\mathrm{E}_{n}(I)$ is the subgroup of $\mathrm{E}_{n}(R)$ generated by elements $e_{i j}(x)$ with $x \in I$. The relative elementary group $\mathrm{E}_{n}(R, I)$ is the normal closure of $\mathrm{E}_{n}(I)$ in $\mathrm{E}_{n}(R)$.

- The group $\operatorname{ESp}_{2 m}(I)$ is the subgroup of $\operatorname{ESp}_{2 m}(R)$ generated by elements $s e_{i j}(x)$ with $x \in I$. The relative elementary symplectic group $\operatorname{ESp}_{2 m}(R, I)$ is the normal closure of $\operatorname{ESp}_{2 m}(I)$ in $\operatorname{ESp}_{2 m}(R)$.

- We say stable range condition $s r_{n}(R)$ holds for $R$ if given $\left(a_{1}, a_{2}, \ldots, a_{n+1}\right) \in \mathrm{Um}_{n+1}(R)$, there exist $c_{i} \in R$ such that $\left(a_{1}+c_{1} a_{n+1}, a_{2}+c_{2} a_{n+1}, \ldots, a_{n}+c_{n} a_{n+1}\right) \in \operatorname{Um}_{n}(R)$.

- The stable range of $R$, denoted by $\operatorname{sr}(R)$, is the least integer $n$ such that $\operatorname{sr}_{n}(R)$ holds. The stable dimension of $R$ is $\operatorname{sdim}(R)=\operatorname{sr}(R)-1$.

- Let $(a, b) \in \operatorname{Um}_{2}(R)$ and $c, d \in R$ be such that $a d-b c=1$. The Mennicke symbol ms $(a, b)$ is defined as follows [9, Chapter 2] :

$$
\operatorname{ms}(a, b)=\text { class of }\left[\begin{array}{lll}
a & b & 0 \\
c & d & 0 \\
0 & 0 & 1
\end{array}\right] \in \mathrm{SL}_{3}(R) / \mathrm{E}_{3}(R) .
$$

Observe that if $\mathrm{ms}\left(a_{1}, b_{1}\right)=\mathrm{ms}\left(a_{2}, b_{2}\right)$, then there exists $\sigma \in \mathrm{SL}_{2}(R) \cap \mathrm{E}_{3}(R)$ such that $\left(a_{1}, b_{1}\right) \sigma=$ $\left(a_{2}, b_{2}\right)$. Further $\operatorname{ms}\left(a a^{\prime}, b\right)=\operatorname{ms}(a, b) \mathrm{ms}\left(a^{\prime}, b\right)$.

- The excision ring $R \oplus I$ has coordinatewise addition. The multiplication is given as $(r, i) \cdot(s, j)=$ $(r s, r j+s i+i j)$, where $r, s \in R$ and $i, j \in I$. The multiplicative identity is $(1,0)$.

We note a result from [12, Proposition 3.1].

Lemma 2.2. Let $A$ be a ring and $R$ be an affine A-algebra of dimension d. If $I$ is an ideal of $R$, then $R \oplus I$ is also an affine A-algebra of dimension d.

Definition 2.3. A ring homomorphism $\phi: B \rightarrow D$ has a section if there exists a ring homomorphism $\gamma: D \hookrightarrow$ $B$ so that $\phi \circ \gamma$ is the identity on $D$. In this case we call $D$ to be a retract of $B$.

The following lemma is due to Suslin and is an easy consequence of [9] Lemma 4.3, Chapter 3]. 
Lemma 2.4. Let $B, D$ be rings and $\pi: B \rightarrow D$ has a section. If $J=\operatorname{ker}(\pi)$, then $\mathrm{E}_{n}(B, J)=\mathrm{E}_{n}(B) \cap$ $\mathrm{SL}_{n}(B, J)$ for $n \geq 3$.

Remark 2.5. Let $R$ be a ring and $I$ be an ideal of $R$. There is a natural homomorphism $\omega: R \oplus I \rightarrow R$ given by $(x, i) \mapsto x+i \in R$. The map $\gamma: R \rightarrow R \oplus I$ given by $x \mapsto(x, 0)$ is a section of $\omega$. Further, $(R \oplus I) /(0 \oplus I) \simeq R$ via the projection map $\pi: R \oplus I \longrightarrow R$.

Let $v=\left(1+i_{1}, i_{2}, \ldots, i_{n}\right) \in \operatorname{Um}_{n}(R, I)$, where $i_{j}$ 's are in $I$. Then $\tilde{v}=\left(\left(1, i_{1}\right),\left(0, i_{2}\right), \ldots,\left(0, i_{n}\right)\right) \in$ $\mathrm{Um}_{n}(R \oplus I, 0 \oplus I)$. We call $\tilde{v}$ to be a lift of $v$. Note that $\omega$ sends $\tilde{v}$ to $v$.

We state two results from [21, Corollary 17.3, Corollary 18.1, Theorem 18.2].

Theorem 2.6. Let $R$ be an affine $C$-algebra of dimension $d \geq 2$, where $C$ is either a subfield $F$ of $\overline{\mathbb{F}}_{p}$ or $C=\mathbb{Z}$. Then

- If $d=2$, then $\mathrm{E}_{3}(R)$ acts transitively on $\mathrm{Um}_{3}(R)$.

- If $d \geq 3$, then $\operatorname{sr}(R) \leq d$. As a consequence, $\mathrm{E}_{d+1}(R)$ acts transitively on $\operatorname{Um}_{d+1}(R)$.

Corollary 2.7. Let $R$ be an affine algebra of dimension $d \geq 2$ over a subfield $F$ of $\overline{\mathbb{F}}_{p}$ and $I$ be an ideal of $R$. Then $\mathrm{E}_{d+1}(R, I)$ acts transitively on $\operatorname{Um}_{d+1}(R, I)$.

Proof : Let $v \in \operatorname{Um}_{d+1}(R, I)$. In view of Lemma 2.2, $R \oplus I$ is an affine algebra of dimension $d$ over $F$. By theorem 2.6, there exists $\varepsilon_{1} \in \mathrm{E}_{d+1}(R \oplus I)$ such that $\tilde{v} \varepsilon_{1}=e_{1}$. Going modulo $0 \oplus I$, we have $e_{1} \overline{\varepsilon_{1}}=e_{1}$ with $\overline{\varepsilon_{1}} \in \mathrm{E}_{d+1}(R)$. Now replacing $\varepsilon_{1}$ by $\varepsilon_{1}\left(\bar{\varepsilon}_{1}\right)^{-1}$ and using Lemma 2.4 we may assume that $\varepsilon_{1} \in \mathrm{E}_{d+1}(R \oplus I, 0 \oplus I)$ satisfying $\tilde{v} \varepsilon_{1}=e_{1}$. Now applying $\omega$, defined in Remark 2.5, to last equation we get $v \varepsilon=e_{1}$ for some $\varepsilon \in \mathrm{E}_{d+1}(R, I)$.

Next we note a result of Vaserstein [21, Lemma 5.5].

Lemma 2.8. Let $R$ be a ring. Then for any $m \geq 1, \mathrm{E}_{2 m}(R) e_{1}=\left(\operatorname{Sp}_{2 m}(R) \cap \mathrm{E}_{2 m}(R)\right) e_{1}$.

Remark 2.9. It was observed in [3, Lemma 2.13] that Vaserstein's proof actually shows that $\mathrm{E}_{2 m}(R) e_{1}=$ $\operatorname{ESp}_{2 m}(R) e_{1}$. The relative version was proved in [3, Theorem 5.5] (when $2 R=R$ ) and in [8, Theorem 3.9] in general.

Theorem 2.10. Let $R$ be a ring and $I$ be an ideal of $R$. If $v \in \operatorname{Um}_{2 n}(R, I)$, then $v \mathrm{E}_{2 n}(R, I)=v \mathrm{ESp}_{2 n}(R, I)$ for $n \geq 2$.

Corollary 2.11. Let $R$ be an affine algebra of dimension 3 over a subfield $F$ of $\overline{\mathbb{F}}_{p}$ which $2 R=R$ and $I$ be an ideal of $R$. Then $\operatorname{ESp}_{4}(R, I)$ acts transitively on $\operatorname{Um}_{4}(R, I)$.

Proof : It follows from theorem 2.10 and corollary 2.7

Vaserstein [20] showed that the natural map $\phi_{n, n+1}: \operatorname{Sp}_{2 n}(R) / \operatorname{ESp}_{2 n}(R) \longrightarrow \operatorname{Sp}_{2 n+2}(R) / \operatorname{ESp}_{2 n+2}(R)$ is bijective for $n \geq \operatorname{dim}(R)+2$. The injective stability bound was improved by Rao and Basu [2] and Basu, Chattopadhyay and Rao [4] as follows.

Theorem 2.12. Let $R$ be a smooth affine algebra over a perfect $C_{1}$-field, then the natural map

$$
\phi_{n, n+1}: \operatorname{Sp}_{2 n}(R) / \operatorname{ESp}_{2 n}(R) \longrightarrow \operatorname{Sp}_{2 n+2}(R) / \operatorname{ESp}_{2 n+2}(R)
$$

is injective for $n \geq \operatorname{dim}(R)+1$. 
The next result is due to Suslin-Vaserstein [21, corollary 7.4].

Lemma 2.13. Let $R$ be a commutative ring and $v, v^{\prime} \in \mathrm{Um}_{3}(R)$. If $v \beta=v^{\prime}$ for some $\beta \in \mathrm{SL}_{3}(R) \cap \mathrm{E}_{4}(R)$, then there exists $\beta_{1} \in \mathrm{E}_{3}(R)$ such that $v \beta_{1}=v^{\prime}$.

We note a result of Fasel [5, Lemma 3.3].

Lemma 2.14. Let $S$ be a smooth affine surface over an algebraically closed field of characteristic $\neq 2,3$. Then $\mathrm{SL}_{2}(S) \cap \mathrm{E}(S)=\mathrm{SL}_{2}(S) \cap \mathrm{E}_{3}(S)=\mathrm{SL}_{2}(S) \cap \mathrm{ESp}_{4}(S)=\operatorname{SL}_{2}(S) \cap \operatorname{ESp}(S)$.

The next result is due to Garge and Rao [6, Lemma 3.5] for $n=d+1$,. The same proof works in our case.

Lemma 2.15. Let $R$ be a ring of stable dimension $\leq 2 n-4$ and $n \geq 3$. If the group structure on $\operatorname{Um}_{n}\left(R_{\text {red }}\right) / \mathrm{E}_{n}\left(R_{\text {red }}\right)$ is nice, then the group structure on $\operatorname{Um}_{n}(R) / \mathrm{E}_{n}(R)$ is nice.

Next we note a slightly modified version of pre-stabilization result of van der Kallen [10, Theorem 2.2]. We get this version by applying van der Kallen's result [10, Theorem 2.2] to the matrix $g^{t}$ and then taking the transpose afterwards.

Theorem 2.16. Let $R$ be a ring of stable dimension $\leq 2 n-3$ with $n \geq 3$ and $I$ be an ideal of $R$. Let $i, j \geq 0$ and $g \in \mathrm{GL}_{n+i}(R) \cap \mathrm{E}_{n+i+j+1}(R, I)$. Then there exist matrices $u, v, w, M$ with entries in $I$ and $q$ with entries in $R$ such that

$$
\left[\begin{array}{cc}
I_{i+1}+u q & v \\
w q & I_{n-1}+M
\end{array}\right] \in \mathrm{E}_{n+i}(R, I) \text { and }\left[\begin{array}{cc}
I_{j+1}+q u & q v \\
w & I_{n-1}+M
\end{array}\right] \in g \mathrm{E}_{n+j}(R, I) .
$$

We note a local-global principle for unimodular rows [16, Theorem 2.3].

Theorem 2.17. Let $R$ be a ring, $v \in \operatorname{Um}_{n}(R[X])$ and $n \geq 3$. Suppose that for all $\mathfrak{m} \in$ Maxspec $(R)$, $v \equiv v(0)\left(\bmod \mathrm{E}_{n}\left(R_{\mathfrak{m}}[X]\right)\right.$. Then $v \equiv v(0)\left(\bmod \mathrm{E}_{n}(R[X])\right.$.

The next result of van der Kallen and Rao [15, Theorem 1] is about the drop in injective stability for $\mathrm{SK}_{1}$ in the case of non-singular affine algebra.

Theorem 2.18. Let $R$ be a non-singular affine algebra of dimension $d \geq 2$ over a perfect $C_{1}$-field $k$. Then $\mathrm{SL}_{d+1}(R) \cap \mathrm{E}_{d+2}(R)=\mathrm{E}_{d+1}(R)$ i.e. a stably elementary $\sigma \in \mathrm{SL}_{d+1}(R)$ belongs to $\mathrm{E}_{d+1}(R)$. Consequently, the natural map $\mathrm{SL}_{r}(R) / \mathrm{E}_{r}(R) \longrightarrow S K_{1}(R)$ is an isomorphism for $r \geq d+1$.

\section{MAIN RESULTS}

We start with a lifting lemma for smooth affine algebras over a subfield $F$ of $\overline{\mathbb{F}}_{p}$.

Lemma 3.1. Let $R$ be a three-dimensional smooth affine algebra over a subfield $F$ of $\overline{\mathbb{F}}_{p}$ with $2 R=R$. Let $a \in R$ be such that $\operatorname{dim}(R / a R)=2$ and $R / a R$ is smooth. If $\alpha \in \mathrm{SL}_{2}(R / a R) \cap \mathrm{ESp}(R / a R)$, then there exists $\beta \in \mathrm{SL}_{2}(R) \cap \mathrm{E}(R)$ such that $\alpha \equiv \beta \bmod a R$. 
Proof : By stabilization theorem 2.12, $\alpha \in \mathrm{ESp}_{6}(R / a R)$. Therefore there exists $\beta^{\prime} \in \mathrm{ESp}_{6}(R)$ such that

$$
\beta^{\prime} \bmod a R=\left[\begin{array}{cc}
I_{4} & 0 \\
0 & \alpha
\end{array}\right] .
$$

In view of Suslin-Vaserstein's estimates and theorem 2.10, $\operatorname{ESp}_{6}(R, a R)$ acts transitively on $\operatorname{Um}_{6}(R, a R)$. Therefore by altering $\beta^{\prime}$ we may assume that its first row is $(1,0,0,0,0,0)$. By the symplectic condition,

$$
\beta^{\prime}=\left[\begin{array}{ccc}
1 & 0 & 0 \\
* & 1 & * \\
* & 0 & \beta^{\prime \prime}
\end{array}\right]
$$

for some $\beta^{\prime \prime} \in \mathrm{SL}_{4}(R)$. Now observe that $e_{1} \beta^{\prime \prime} \in \mathrm{Um}_{4}(R, a R)$. In view of corollary 2.11 $\operatorname{ESp}_{4}(R, a R)$ acts transitively on $\operatorname{Um}_{4}(R, a R)$. Therefore by altering $\beta^{\prime}$ by an elementary symplectic matrix we may assume that the first row of $\beta^{\prime \prime}$ is $(1,0,0,0)$. Now by the symplectic condition,

$$
\beta^{\prime}=\left[\begin{array}{lllll}
1 & 0 & 0 & 0 & 0 \\
* & 1 & * & * & * \\
* & 0 & 1 & 0 & 0 \\
* & 0 & * & 1 & * \\
* & 0 & * & 0 & \beta
\end{array}\right]
$$

and $\beta$ satisfies our requirements.

Lemma 3.2. Let $R$ be an affine algebra of dimension $d \geq 4$ over a subfield $F$ of $\overline{\mathbb{F}}_{p}$ with $2 R=R$ and $\left(a_{1}, \ldots, a_{d}\right) \in \operatorname{Um}_{d}(R)$. Let $B=R /\left(a_{4} R+\cdots+a_{d} R\right)$ and $C=R /\left(a_{3} R+\cdots+a_{d} R\right)$. Assume that $B$ and $C$ are smooth affine algebras over $F$ with $\operatorname{dim}(B) \leq 3$ and $\operatorname{dim}(C) \leq 2$. Let $\alpha \in \mathrm{SL}_{2}(C) \cap \operatorname{ESp}(C)$ and $\left(\overline{a_{1}}, \overline{a_{2}}\right) \cdot \alpha=\left(\overline{b_{1}}, \overline{b_{2}}\right)$ for some $b_{1}, b_{2} \in R$. Then there exists $\gamma \in \mathrm{E}_{d}(R)$ such that $\left(a_{1}, \ldots, a_{d}\right) \gamma=$ $\left(b_{1}, b_{2}, a_{3}, \ldots, a_{d}\right)$.

Proof : In view of lemma 3.1, there exists $\beta^{\prime} \in \mathrm{SL}_{2}(B) \cap \mathrm{E}(B)$ such that $\left(\overline{a_{1}}, \overline{a_{2}}, \overline{a_{3}}\right) \cdot\left(\beta^{\prime} \perp 1\right)=$ $\left(\overline{b_{1}+a_{3} u_{3}}, \overline{b_{2}+a_{3} v_{3}}, \overline{a_{3}}\right)$ for some $\overline{u_{3}}, \overline{v_{3}} \in B$. Using elementary transformations, we can find a $\beta \in$ $\mathrm{SL}_{3}(B) \cap \mathrm{E}(B)$ such that $\left(\overline{a_{1}}, \overline{a_{2}}, \overline{a_{3}}\right) \cdot \beta=\left(\overline{b_{1}}, \overline{b_{2}}, \overline{a_{3}}\right)$. By theorem $2.18, \beta \in \mathrm{SL}_{3}(B) \cap \mathrm{E}_{4}(B)$. Therefore by lemma 2.13, there exists $\beta_{1} \in \mathrm{E}_{3}(B)$ such that

$$
\left(\overline{a_{1}}, \overline{a_{2}}, \overline{a_{3}}\right) \cdot \beta_{1}=\left(\overline{b_{1}}, \overline{b_{2}}, \overline{a_{3}}\right) .
$$

Now our desired $\gamma$ can be easily constructed in the form

$$
\gamma=\left[\begin{array}{cc}
\beta_{1}^{\prime} & 0 \\
* & I_{d-3}
\end{array}\right]
$$

where $\beta_{1}^{\prime} \in \mathrm{E}_{3}(R)$ such that $\beta_{1}^{\prime} \equiv \beta_{1} \bmod \left(a_{4} R+\cdots+a_{d} R\right)$.

The proof of next result is similar to [6, Proposition 3.8]. We refer to [6] for the definition of admissible pair and admissible sequence. 
Proposition 3.3. Let $R$ be an affine algebra of dimension $d \geq 5$ over a field $F \subset \overline{\mathbb{F}}_{p}$ with $p>3$. Let $I$ be an ideal of $R$ of height $\geq 1$. Let $(v, w)$ be an admissible pair of size $d$ over $R$. Then there exists another admissible pair $\left(v^{\prime}, w^{\prime}\right)$ of size $d$ over $R$ such that both $v^{\prime}, w^{\prime} \equiv e_{d}(\bmod I)$ and $(v, w) \rightarrow\left(v^{\prime}, w^{\prime}\right)$ is an admissible sequence.

Proof : Let $v=\left(a, a_{1}, \ldots, a_{d-1}\right)$ and $w=\left(b, a_{1}, \ldots, a_{d-1}\right)$ be a pair of admissible rows of size $d$ over $R$. Let bar denote reduction modulo $I$. Let $\bar{v}=\left(\bar{a}, \overline{a_{1}}, \ldots, \overline{a_{d-1}}\right)$ and $\bar{w}=\left(\bar{b}, \overline{a_{1}}, \ldots, \overline{a_{d-1}}\right)$. Since $\bar{v}$ is unimodular, there exist $\bar{f}, \overline{f_{1}}, \ldots, \overline{f_{d-1}} \in \bar{R}$ such that $\bar{a} \bar{f}+\overline{a_{1}} \overline{f_{1}}+\cdots+\overline{a_{d-1}} \overline{f_{d-1}}=1$. In particular, the elements $\bar{a}$ and $\overline{a_{1}} \overline{f_{1}}+\cdots+\overline{a_{d-1}} \overline{f_{d-1}}$ are coprime in $\bar{R}$. Hence, by [21, Lemma 9.2], there exists $\bar{z} \in \bar{R}$ such that $\overline{a^{\prime}}=\bar{a}+\overline{a_{1} z} \overline{f_{1}}+\cdots+\overline{a_{d-1} z \overline{f_{d-1}}}$ is not a zero divisor. Thus $\mathrm{ht}\left(\overline{a^{\prime}}\right) \geq 1$. Similarly, one can find $\bar{y}, \overline{g_{1}}, \ldots, \overline{g_{d-1}} \in \bar{R}$ such that if $\overline{b^{\prime}}=\bar{b}+\overline{a_{1} y g_{1}}+\cdots+\overline{a_{d-1} y g_{d-1}}$, then $\operatorname{ht}\left(\overline{b^{\prime}}\right) \geq 1$. Therefore ht $\left(\overline{a^{\prime} b^{\prime}}\right) \geq 1$. Let $\overline{v_{1}}=\left(\overline{a^{\prime}}, \overline{a_{1}}, \ldots, \overline{a_{d-1}}\right)$ and $\overline{w_{1}}=\left(\overline{b^{\prime}}, \overline{a_{1}}, \ldots, \overline{a_{d-1}}\right)$. Then $(\bar{v}, \bar{w}) \rightarrow\left(\overline{v_{1}}, \overline{w_{1}}\right)$ is an admissible sequence.

Let $\overline{\bar{R}}$ denotes reduction modulo $\left(\overline{a^{\prime} b^{\prime}}\right)$ in $\bar{R}$. Note that $\left(\overline{\overline{a_{1}}}, \ldots, \overline{\overline{a_{d-1}}}\right) \in \operatorname{Um}_{d-1}(\overline{\bar{R}})$. Since $\operatorname{dim}(\overline{\bar{R}}) \leq d-2$ and $d-2 \geq 3$, by theorem $2.6 \operatorname{sr}(\overline{\bar{R}}) \leq d-2$. Therefore $\operatorname{sr}_{d-2}(\overline{\bar{R}})$ holds. Thus there exist $\overline{\overline{c_{i}}} \in \overline{\bar{R}}$ for $1 \leq i \leq$ $d-2$ such that if $\overline{\overline{a_{i}^{\prime}}}=\overline{\overline{a_{i}}}+\overline{\overline{c_{i} a_{d-1}}}$ then $\left(\overline{\overline{a_{1}^{\prime}}}, \ldots, \overline{\overline{a_{d-2}^{\prime}}}\right) \in \operatorname{Um}_{d-2}(\overline{\bar{R}})$. Thus $\left(\overline{a^{\prime}}, \overline{a_{1}^{\prime}}, \ldots, \overline{a_{d-2}^{\prime}}\right) \in \operatorname{Um}_{d-1}(\bar{R})$ and $\left(\overline{b^{\prime}}, \overline{a_{1}^{\prime}}, \ldots, \overline{a_{d-2}^{\prime}}\right) \in \operatorname{Um}_{d-1}(\bar{R})$.

Summarizing the above discussion, we can modify the unimodular rows $\bar{v}$ and $\bar{w}$ to the unimodular rows $\overline{v_{2}}=\left(\overline{a^{\prime}}, \overline{a_{1}^{\prime}}, \ldots, \overline{a_{d-2}^{\prime}}, \overline{a_{d-1}}\right)$ and $\overline{w_{2}}=\left(\overline{b^{\prime}}, \overline{a_{1}^{\prime}}, \ldots, \overline{a_{d-2}^{\prime}}, \overline{a_{d-1}}\right)$ such that $\left(\overline{a^{\prime}}, \overline{a_{1}^{\prime}}, \ldots, \overline{a_{d-2}^{\prime}}\right) \in \operatorname{Um}_{d-1}(\bar{R})$ and $\left(\overline{b^{\prime}}, \overline{a_{1}^{\prime}}, \ldots, \overline{a_{d-2}^{\prime}}\right) \in \operatorname{Um}_{d-1}(\bar{R})$. Then $(\bar{v}, \bar{w}) \rightarrow\left(\overline{v_{1}}, \overline{w_{1}}\right) \rightarrow\left(\overline{v_{2}}, \overline{w_{2}}\right)$ is an admissible sequence. Now follow the proof of [6, Proposition 3.8] to complete the proof.

The proof of next result follows that of [6, Theorem 3.9]. We will only mention the necessary changes.

Theorem 3.4. Let $R$ be an affine algebra of dimension $d \geq 5$ over $\overline{\mathbb{F}}_{p}$ with $p>3$. Then the abelian group structure on the orbit space $\mathrm{Um}_{d}(R) / \mathrm{E}_{d}(R)$ is nice.

Proof : In view of lemma 2.15, we may assume that $R$ is reduced. Let $v=\left(a, a_{1}, \ldots, a_{d-1}\right), w=$ $\left(b, a_{1}, \ldots, a_{d-1}\right)$.

(1) Let $J$ be the ideal defining the singular locus of $R$. Since $R$ is a reduced, $\operatorname{ht}(J) \geq 1$. By proposition 3.3 we may assume $(v, w)$ to be an admissible pair such that $v, w \equiv e_{d}(\bmod J)$.

(2) Applying Swan's version of Bertini [19] (see also [13, P. 413]), we can add a linear combination of $a b, a_{1}, \ldots, a_{d-2}$ to $a_{d-1}$ to get $a_{d-1}^{\prime}=a_{d-1}+\lambda a b+\sum_{i=1}^{d-2} \lambda_{i} a_{i}$ for $\lambda, \lambda_{i} \in R$ and $R /\left(a_{d-1}^{\prime}\right)$ is smooth outside the singular set of $R$. Since $a_{d-1}^{\prime} \equiv 1(\bmod J), R /\left(a_{d-1}^{\prime}\right)$ is smooth of dimension atmost $d-1$.

Now with $b_{i}=a_{i}+\sum_{j=1}^{i-1} \lambda_{j i} a_{j}+\mu_{i} a b$ for suitable $\mu_{i}, \lambda_{j i} \in R$, we have an admissible sequence $(v, w) \rightarrow\left(v^{\prime}, w^{\prime}\right)$ where $v^{\prime}=\left(a, b_{1}, \ldots, b_{d-2}, a_{d-1}^{\prime}\right), w^{\prime}=\left(b, b_{1}, \ldots, b_{d-2}, a_{d-1}^{\prime}\right)$ and $\operatorname{ht}\left(b_{2}, \ldots, b_{d-2}, a_{d-1}^{\prime}\right) \geq d-2, \operatorname{ht}\left(b_{3}, \ldots, b_{d-2}, a_{d-1}^{\prime}\right) \geq d-3$. Note that $v^{\prime}, w^{\prime} \equiv e_{d}(\bmod J)$. We also have $\operatorname{dim}\left(R /\left(b_{2}, \ldots, b_{d-2}, a_{d-1}^{\prime}\right)\right) \leq 2$ and $\operatorname{dim}\left(R /\left(b_{3}, \ldots, b_{d-2}, a_{d-1}^{\prime}\right)\right) \leq 3$. By Swan's version of Bertini as in [19], $R /\left(b_{2}, \ldots, b_{d-2}, a_{d-1}^{\prime}\right)$ and $R /\left(b_{3}, \ldots, b_{d-2}, a_{d-1}^{\prime}\right)$ are smooth outside the singular set of $R$. Since $a_{d-1}^{\prime} \equiv 1(\bmod J)$, both $R /\left(b_{2}, \ldots, b_{d-2}, a_{d-1}^{\prime}\right)$ and $R /\left(b_{3}, \ldots, b_{d-2}, a_{d-1}^{\prime}\right)$ are smooth. Note that $[v]=\left[v^{\prime}\right]$ and $[w]=\left[w^{\prime}\right]$ 
(3) Now van der Kallen's product formula in the group $\operatorname{Um}_{d}(R) / \mathrm{E}_{d}(R)$ shows that

$$
[v] *[w]=\left[v^{\prime}\right] *\left[w^{\prime}\right]=\left[\left(a(b+p)-1,(b+p) b_{1}, \ldots, b_{d-2}, a_{d-1}^{\prime}\right)\right]
$$

where $p$ is choosen so that $a p-1$ belongs to the ideal generated by $b_{1}, \ldots, b_{d-2}, a_{d-1}^{\prime}$. Let 'overline' denote the image in $R /\left(b_{2}, \ldots, b_{d-2}, a_{d-1}^{\prime}\right) R$. If 'ms' denote the Mennicke symbol, then

$$
\begin{aligned}
\operatorname{ms}\left(\bar{a}(\bar{b}+\bar{p})-1,(\bar{b}+\bar{p}) \bar{b}_{1}\right) & =\operatorname{ms}(\bar{a}(\bar{b}+\bar{p})-1,(\bar{b}+\bar{p})) \operatorname{ms}\left(\bar{a}(\bar{b}+\bar{p})-1, \bar{b}_{1}\right) \\
& =\operatorname{ms}\left(\bar{a}(\bar{b}+\bar{p})-1, \bar{b}_{1}\right) .
\end{aligned}
$$

Therefore there is a $\bar{\sigma} \in \mathrm{SL}_{2}(\bar{R}) \cap \mathrm{E}_{3}(\bar{R})$ such that

$$
\left(\bar{a}(\bar{b}+\bar{p})-1,(\bar{b}+\bar{p}) \bar{b}_{1}\right) \bar{\sigma}=\left(\bar{a}(\bar{b}+\bar{p})-1, \bar{b}_{1}\right) .
$$

Therefore by lemma 2.14, $\bar{\sigma} \in \operatorname{ESp}(\bar{R})$. Now in view of lemma 3.2, there exists $\gamma \in E_{d}(R)$ such that

$$
\left(a(b+p)-1,(b+p) b_{1}, b_{2}, \ldots, b_{d-2}, a_{d-1}^{\prime}\right) \gamma=\left(a(b+p)-1, b_{1}, b_{2}, \ldots, b_{d-2}, a_{d-1}^{\prime}\right) .
$$

Since $a p-1$ belongs to the ideal generated by $b_{1}, \ldots, b_{d-2}, a_{d-1}^{\prime}$, we have

$$
\begin{aligned}
{\left[v^{\prime}\right] *\left[w^{\prime}\right] } & =\left[\left(a(b+p)-1,(b+p) b_{1}, b_{2}, \ldots, b_{d-2}, a_{d-1}^{\prime}\right)\right] \\
& =\left[\left(a(b+p)-1, b_{1}, b_{2}, \ldots, b_{d-2}, a_{d-1}^{\prime}\right)\right] \\
& =\left[\left(a b, b_{1}, b_{2}, \ldots, b_{d-2}, a_{d-1}^{\prime}\right)\right] \\
& =\left[\left(a b, a_{1}, a_{2}, \ldots, a_{d-2}, a_{d-1}^{\prime}\right)\right] \\
& =\left[\left(a b, a_{1}, a_{2}, \ldots, a_{d-2}, a_{d-1}\right)\right]
\end{aligned}
$$

This proves the required product formula.

Corollary 3.5. Let $R$ be an affine algebra of dimension $d \geq 5$ over $\overline{\mathbb{F}}_{p}$ with $p>3$. If $\sigma \in \operatorname{SL}_{d}(R) \cap \mathrm{E}_{d+1}(R)$, then $e_{1} \sigma$ can be completed to an elementary matrix.

Proof : In view of theorem 2.16, we have

$$
[\sigma]=\left[\begin{array}{cc}
1+u x & u y \\
z^{t} & M
\end{array}\right],[\varepsilon]=\left[\begin{array}{cc}
1+u x & y \\
u z^{t} & M
\end{array}\right],
$$

for some $u, x \in R, y, z \in M_{1, d-1}(R), M \in M_{d-1, d-1}(R), \varepsilon \in E_{d}(R)$. Since the group structure on $\mathrm{Um}_{d}(R) / \mathrm{E}_{d}(R)$ is nice, we have

$$
\left[e_{1} \sigma\right]=[1+u x, u y]=[1+u x, u] *[1+u x, y]=[1+u x, y]=\left[e_{1}\right] .
$$

The last equality $[1+u x, y]=\left[e_{1}\right]$ follows from the existance of $\varepsilon$. 


\section{A NICE GROUP STRUCTURE OVER POLYNOMIAL RINGS}

In [6], Garge and Rao proved that if $R$ is a local ring of dimension $d$ with $2 R=R$, then the group structure on $\mathrm{Um}_{d+1}(R[X]) / \mathrm{E}_{d+1}(R[X])$ is nice. In this section we study the group structure on $\operatorname{Um}_{d+1}(R[X]) / \mathrm{E}_{d+1}(R[X])$, where $R$ is a ring of dimension $d \geq 2$ such that $\mathrm{E}_{d+1}(R)$ acts transitively on $\operatorname{Um}_{d+1}(R)$.

Theorem 4.1. Let $R$ be a ring of dimension $d \geq 2$ with $2 R=R$ such that $\mathrm{E}_{d+1}(R)$ acts transitively on $\operatorname{Um}_{d+1}(R)$. Then

(1) the group structure on $\operatorname{Um}_{d+1}(R[X]) / \mathrm{E}_{d+1}(R[X])$ is nice.

(2) If $\sigma \in \mathrm{SL}_{d+1}(R[X]) \cap \mathrm{E}_{d+2}(R[X])$, then $\left[e_{1} \sigma\right]=\left[e_{1}\right]$.

Proof : (1) Let $[u(X)]=\left[\left(a b, a_{1}, \ldots, a_{d}\right)\right]-\left[\left(a, a_{1}, \ldots, a_{d}\right)\right]-\left[\left(b, a_{1}, \ldots, a_{d}\right)\right]$. For every $\mathfrak{m} \in \operatorname{Maxspec}(R)$, by [6, Theorem 5.1], $[u(X)]_{\mathfrak{m}}=\left[e_{1}\right]$. Therefore by local-global principle 2.17 $[u(X)]=[u(0)]$. Note that $u(0) \in \operatorname{Um}_{d+1}(R)$, by hypothesis of the theorem $[u(0)]=\left[e_{1}\right]$. Thus $[u(X)]=\left[e_{1}\right]$, i.e.

$$
\left[\left(a, a_{1}, \ldots, a_{d}\right)\right] *\left[\left(b, a_{1}, \ldots, a_{d}\right)\right]=\left[\left(a b, a_{1}, \ldots, a_{d}\right)\right] .
$$

(2) Let $\mathfrak{m} \in \operatorname{Maxspec}(R)$. In view of [6, Corollary 5.17], $\left[e_{1} \sigma\right]_{\mathfrak{m}}=\left[e_{1}\right]$. By local-global principle 2.17, we have $\left[e_{1} \sigma\right]=\left[e_{1} \sigma(0)\right]$. Since $e_{1} \sigma(0) \in \operatorname{Um}_{d+1}(R),\left[e_{1} \sigma(0)\right]=\left[e_{1}\right]$. Thus $\left[e_{1} \sigma\right]=\left[e_{1}\right]$.

Lemma 4.2. Let $R$ be a ring of dimension $d \geq 2$ and height of the Jacobson radical $J(R) \geq 1$. Then $\mathrm{E}_{d+1}(R)$ acts transitively on $\operatorname{Um}_{d+1}(R)$.

Proof : Let $v \in \operatorname{Um}_{d+1}(R)$ and $\bar{R}=R / J(R)$. Since $\operatorname{dim}(\bar{R}) \leq d-1$, by Bass [1], there exists $\bar{\varepsilon} \in$ $\mathrm{E}_{d+1}(\bar{R})$ such that $\overline{v \varepsilon}=\overline{e_{1}}$. Let $\varepsilon \in \mathrm{E}_{d+1}(R)$ be a lift of $\bar{\varepsilon}$. Then one has $v \varepsilon=\left(1+a_{0}, a_{1}, \ldots, a_{d}\right)$ for some $a_{i} \in J(R)$. Note that $1+a_{0}$ is a unit in $R$, thus $[v]=\left[e_{1}\right]$.

Next we note a result of Rao from [14, Corollary 2.5].

Lemma 4.3. Let $R$ be a ring of dimension $d \geq 2$ with $d ! R=R$. Then every $v(X) \in \operatorname{Um}_{d+1}(R[X])$ is extended from R, i.e. $v(X) \underset{\mathrm{SL}_{d+1}(R[X])}{\sim} v(0)$.

Corollary 4.4. Let $R$ be a ring of dimension $d \geq 2$ with $d ! R=R$ and $h t(J(R)) \geq 1$. Then every $v(X) \in$ $\mathrm{Um}_{d+1}(R[X])$ is completable.

Proof : In view of lemma 4.3, $v(X) \underset{\mathrm{SL}_{d+1}(R[X])}{\sim} v(0)$. Since $v(0) \in \mathrm{Um}_{d+1}(R)$, by lemma 4.2 $v(0)$ is elementarily completable. Thus $v(X)$ is completable.

Corollary 4.5. Let $R$ be a ring of dimension $d \geq 4$ with $2 R=R$. Assume that either $R$ is an affine $C$-algebra with $C=\mathbb{Z}$ or $C$ is a subfield $F$ of $\overline{\mathbb{F}}_{p}$ or $h t(J(R)) \geq 1$. Then

(1) The group structure on $\operatorname{Um}_{d+1}(R[X]) / \mathrm{E}_{d+1}(R[X])$ is nice.

(2) Let $\sigma \in \mathrm{SL}_{d+1}(R[X]) \cap \mathrm{E}_{d+2}(R[X])$. Then $\left[e_{1} \sigma\right]=\left[e_{1}\right]$.

Proof : (1) Follows from theorem 4.1, lemma 4.2 and theorem 2.6 (2) Follows from theorem 4.1 
Acknowledgement: We thank the referee for insisting to clarify the proof of Prop 3.3. which made some points clearer. We also thank the referee for going through the manuscript with great care. A detailed list of suggestions by the referee improved the exposition considerably.

\section{REFERENCES}

[1] H. Bass; Algebraic K-theory, Benjamin, New York (1968).

[2] R. Basu, R.A. Rao; Injective Stability for $K_{1}$ of Classical Modules, Journal of Algebra 323 (2010), No. 4, 867-877.

[3] P. Chattopadhyay, R.A. Rao; Elementary symplectic orbits and improved $K_{1}$-stability, Journal of K-Theory 7 (2011), $389-403$.

[4] R. Basu, P. Chattopadhyay, R.A. Rao; Some remarks on symplectic injective stability, Proceeding of AMS, 139 (2011), no. 7, 23172325.

[5] J. Fasel; Mennicke symbols, K-cohomology and a Bass-Kubota theorem, Transactions of AMS, 361 (2015), No. 1, 191-208.

[6] A. Garge, R.A. Rao; A nice group structure on the orbit space of unimodular rows, $K$-Theory 38 (2008), no. 2, 113-133.

[7] A. Gupta, A. Garge, R.A. Rao; A nice group structure on the orbit space of unimodular rows-II, J. Algebra 407 (2014), 201-223.

[8] A. Gupta; Optimal injective stability for the symplectic $K_{1} S p$ group, J. Pure Appl. Algebra 219 (2015), no. 5, 1336-1348.

[9] S.K. Gupta, M.P. Murthy; Suslin's work on linear groups over polynomial rings and Serre problem, Indian Statistical Institute, New Delhi.

[10] W. van der Kallen; A module structure on certain orbit sets of unimodular rows, J. Pure Appl. Algebra 57 (1989), 657-663.

[11] W. van der Kallen; From Mennicke symbols to Euler class groups, Algebra, arithmetic and geometry, Part I, II (Mumbai, 2000), 341-354, Tata Inst. Fund. Res. Stud. Math., 16, Bombay, (2002).

[12] M.K. Keshari; Cancellation problem for projective modules over affine algebras, J. K-Theory 3 (2009), 561-581.

[13] M.P. Murthy; Zero cycles and projective modules, Ann. Math. 140 (1994) 405-434.

[14] R.A. Rao; The Bass-Quillen conjecture in dimension three but characteristic $\neq 2,3$ via a question of A. Suslin, Invent. Math. 93 (1988), no. 3, 609-618.

[15] R.A. Rao, W. van der Kallen; Improved stability for $S K_{1}$ and $W M S_{d}$ of a non-singular affine algebra, K-Theory (Strasbourg, 1992). Astérisque no. 226 (1994), 411-420.

[16] R.A. Rao; An elementary transformation of a special unimodular vector to its top coefficient vector, Proceedings of AMS, 93 (1985), no. 1, 21-24.

[17] R.A. Rao, S. Jose; A group structure on squares, Proceedings of AMS, 136 (2008), no. 4, 1181-1191.

[18] A.A. Suslin; On the structure of special linear group over polynomial rings, Math. USSR. Izv. 11 (1977), 221-238.

[19] R.G. Swan; A cancellation theorem for projective modules in metastable range, Invent. Math. 27 (1974), 23-43.

[20] L.N. Vaserstein; On the stablization of Unitary and Orthogonal Groups over a Ring with Involution, Math. Sbornik 81 (123) (1970), No. 3, 307-326.

[21] L.N. Vaserstein, A.A. Suslin; Serre's problem on projective modules, Math. USSR Izv. 10 (1976), no. 5, 937-1001.

[22] C.A. Weibel; Complete intersection points on affine varieties, Comm. Algebra 12 (23-24), 3011-3051.

Manoj K. Keshari, Department of Mathematics, IIT Bombay Mumbai 400076, INDiA

E-mail: Manoj K. Keshari <keshari@math.iitb.ac.in>

Sampat Sharma, Department of Mathematics, IIT Bombay Mumbai 400076, INDiA

E-mail: Sampat Sharma<sampat@math.iitb.ac.in; sampat.iisermegmail.com> 